\title{
Fatal neonatal lactic acidosis caused by a novel de novo mitochondrial G7453A tRNA-Serine ${ }^{(\mathrm{UCN})}$ mutation
}

\author{
Alexandra Götz' ${ }^{1}$ Pirjo Isohanni ${ }^{1,2}$, Brita Liljeström³ ${ }^{3}$ Jaana Rummukainen ${ }^{4}$, Kari Nikolajev ${ }^{5}$, Eila Herrgård ${ }^{5}$, Sanna Marjavaara' \\ and Anu Suomalainen ${ }^{1,6}$
}

INTRODUCTION: Heteroplasmic mitochondrial DNA (mtDNA) mutations are an important cause of childhood disorders, but the role of homoplasmic mtDNA mutations in severe neonatal manifestations is not well understood.

METHODS: The following were performed: full mtDNA sequencing for mutation detection, blue-native protein analysis of autopsy-derived tissues to detect respiratory chain (RC) deficiency, light and electron microscopy for morphologic analysis, and northern blot and computational modeling to study the effect of mtDNA mutations on transfer RNA (tRNA) stability.

RESULTS: We describe data from a patient with fatal neonatal lactic acidosis caused by a novel homoplasmic mutation at a highly conserved nucleotide G7453A within the tRNA ${ }^{\text {Ser (UCN) }}$ in mtDNA. The patient's heart, skeletal muscle, brain, and liver showed severe combined complex I and IV (Cl and CIV) deficiencies, accompanied by severe depletion of mature tRNA $A^{\text {Ser }(U C N)}$. The mutation was absent in the patient's mother and in a placental sample from a subsequent pregnancy of the mother, suggesting a de novo mutation.

DISCUSSION: We conclude that the G7453A mutation of mtDNA manifests with exceptional severity as compared with other tRNA ${ }^{\mathrm{Ser}(U C N)}$ mutations, typically associated with sensorineural deafness. De novo homoplasmic mtDNA tRNAmutations should be considered as a cause of fatal neonatal lactic acidosis.

M itochondrial dysfunction is a common cause of severe childhood disorders, sometimes manifesting as early as in the neonatal period. Mitochondrial diseases are most often caused by dysfunction of the respiratory chain (RC), the only system in the human body under a dual genomic control: mitochondrial genome (mtDNA) of $16,569 \mathrm{bp}$ and nuclear genome. mtDNA is a circular molecule that encodes 13 of the $\sim 90$ proteins of the RC (1), as well as transfer RNAs (tRNAs) and ribosomal RNAs (rRNAs) required for their translation. mtDNA is maternally inherited, and therefore mtDNA point mutations are typically inherited from the mother by all her offspring.

The main function of the $\mathrm{RC}$ in mitochondria is to participate in the generation of adenine triphosphate (ATP), the general energy currency of the cell. ATP synthesis occurs in the oxidative phosphorylation system, which is composed of RC enzyme complexes I-IV (CI-CIV), and the ATP synthase. The $\mathrm{RC}$ couples the sequential transfer of electrons from reduced electron carriers nicotinamide and flavin adenine dinucleotides to molecular oxygen, with vectorial proton transfer across the membrane. This generates an electrochemical proton gradient across the inner membrane, and this is released through ATP synthase to phosphorylate adenosine diphosphate to ATP. A defect in the RC causes a deficiency in ATP generation.

Epidemiological studies have shown that the frequency of disorders caused by $\mathrm{mtDNA}$ has been underestimated; mtDNA diseases have been reported to occur at a frequency of $1 / 3,600$ $1 / 7,000$ depending on the population $(2,3)$, and specific pathogenic mutations have been detected in cord blood samples in $1 / 200$ live births (4). Mitochondrial diseases in infants are heterogeneous, typically multisystemic, and result in early death. Often, no molecular diagnosis is made. mtDNA mutations affecting tRNA genes have been shown to be a common cause of human disease (5), but neonatal manifestations are rare and probably underdiagnosed. Pathogenic tRNA mutations are typically heteroplasmic, because homoplasmy is considered incompatible with life. However, some reports of infants with homoplasmic mtDNA mutations have been reported, e.g., in NARP/MILS syndrome (neuropathy, ataxia, retinitis pigmentosa/maternally inherited Leigh syndrome) $(6,7)$.

\section{RESULTS}

We identified a novel homoplasmic point mutation, G7453A in the tRNA ${ }^{\text {Ser (UCN) }}$ gene of mtDNA, in the patient's skeletal muscle sample (Figure 1a). However, the mother had no detectable mutant mtDNA in her blood, and a placental sample of a subsequent pregnancy in the mother also showed no mutant mtDNA. The mutation was not reported in mtDNA variant databases (MITOMAP: A human mitochondrial genome database, http:// www.mitomap.org/MITOMAP; mtDB - Human Mitochondrial Genome Database, http://www.mtdb.igp.uu.se/) or found in Finnish mtDNAs sequenced in our laboratory $(n=71)$. Realtime quantitative PCR showed that the amount of mtDNA was

\footnotetext{
'Research Programs Unit, Molecular Neurology, Biomedicum-Helsinki, University of Helsinki, Helsinki, Finland; ${ }^{2}$ Department of Gynaecology and Pediatrics, Hospital for Children and Adolescents, Helsinki University Central Hospital, Helsinki, Finland; ${ }^{3}$ Department of Clinical Genetics, University Hospital, Kuopio, Finland; ${ }^{4}$ Department of Pathology, University Hospital, Kuopio, Finland; ${ }^{5}$ Department of Pediatrics, University Hospital, Kuopio, Finland; ${ }^{6}$ Department of Neurology, Helsinki University Central Hospital, Helsinki, Finland. Correspondence: Anu Suomalainen (anu.wartiovaara@helsinki.fi)
} 
a

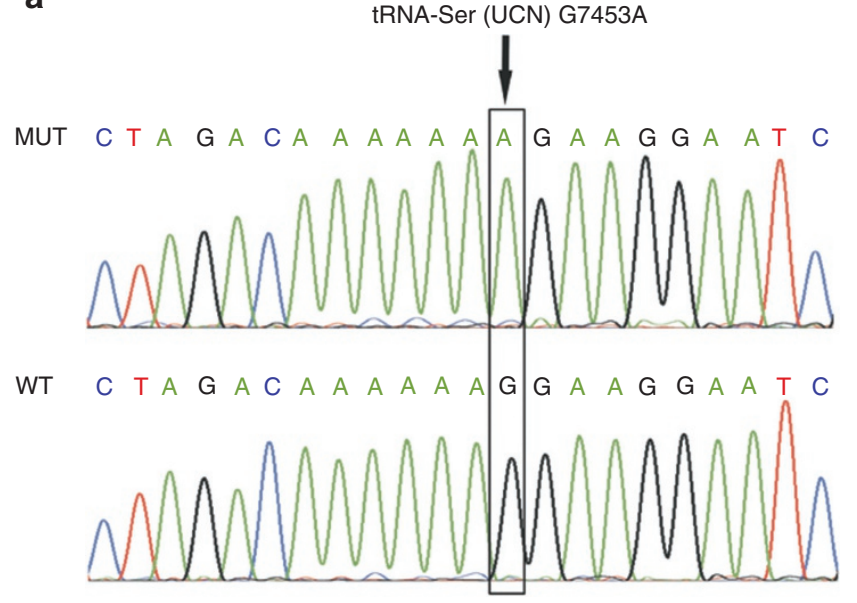

b

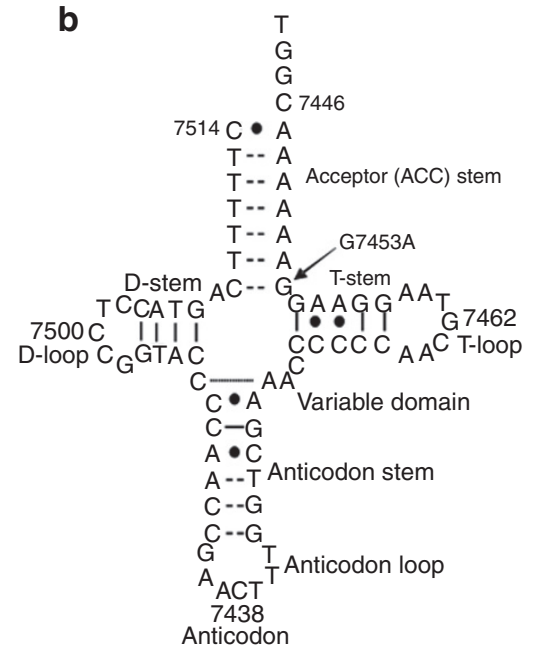

C

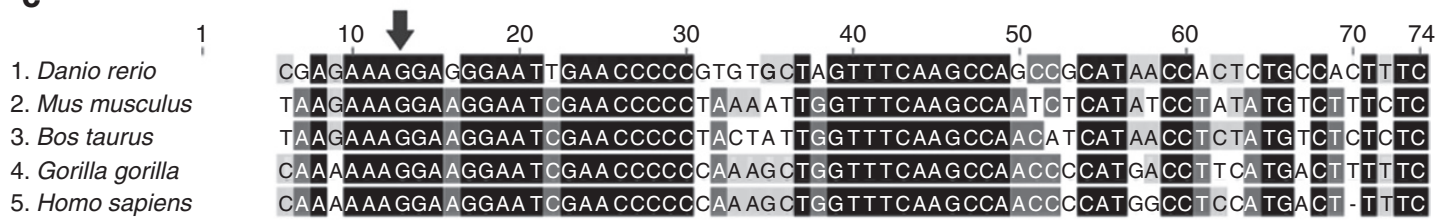

d

$\begin{array}{llllllllll}\mathrm{C} 1 & \mathrm{C} 2 & \mathrm{P} 1 & \mathrm{C} 1 & \mathrm{C} 2 & \mathrm{P} 1 & \mathrm{C} 1 & \mathrm{C} 2 & \mathrm{P} 1\end{array}$

e

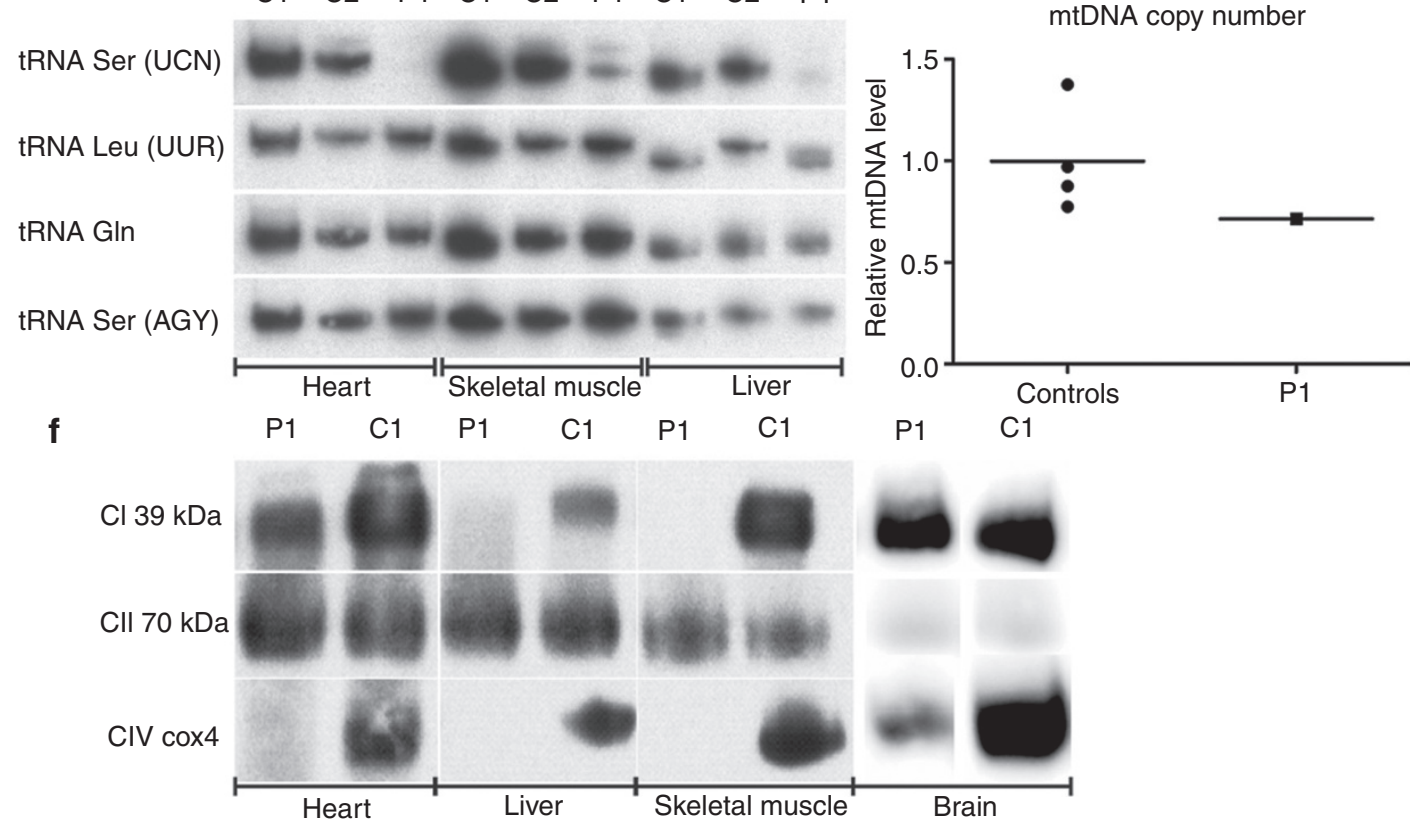

Figure 1. Mitochondrial DNA sequence analysis. (a) Partial mtDNA sequence encoding tRNA ${ }^{\text {Ser (UCN) }}$ showing the homoplasmic $G>A$ nucleotide substitution at position 7453. (b) Cloverleaf structure of tRNA $A^{\text {Ser }(U C N)}$ indicating the position of the $\mathrm{G}>\mathrm{A} 7453$ mutation at the highly conserved nucleotide of the ACC stem. (c) The G7453 nucleotide is highly conserved throughout many species (arrow). Sequence alignment was carried out using the Geneious bioinformatics software for sequence alignment (Geneious v5.1, available from http://www.geneious.com). Sequence similarity is highlighted as follows: black $-100 \%$ identical, dark gray $->80 \%$ similar, light gray $-60-80 \%$ similar, and white $-<60 \%$ similar. (d) Northern blot analysis of RNA from postmortem heart, skeletal muscle, and liver of the patient, and from two controls in whom the cause of death was not related to mitochondrial disease. The ages of the patient (P) and controls (CS) were: $P 1,10 \mathrm{~d} ; C 1,1 \mathrm{y} ; \mathrm{C2}, 2 \mathrm{y}$. Hybridization signals against mitochondrial tRNA $A^{\text {Ser (UCN), }}$ tRNA ${ }^{\text {Leu (UUR) }}$, tRNA $A^{\text {Gln }}$, and tRNA ${ }^{\text {Ser (AGY) }}$ are shown. (e) mtDNA copy number is normal in skeletal muscle samples of the patient, as determined by quantitative real-time PCR and expressed as a ratio of the mitochondrial MT-CYB gene to the nuclear APP gene. Values are shown relative to the average of all four control samples. (f) Blue-native electrophoresis of mitochondrial respiratory chain complexes in the autopsy-derived heart, liver, skeletal muscle, and brain. P1 shows a severely decreased amount of fully assembled complex I (CI) in heart, total $\mathrm{Cl}$ deficiency in the liver and skeletal muscle, and total complex IV (CIV) deficiency in brain, heart, liver, and skeletal muscle. C1: healthy subject. Antibodies against CI $39 \mathrm{kDa}$, CII $70 \mathrm{kDa}$, and CIV cytochrome c oxidase subunit 4. ACC stem, acceptor stem; mtDNA, mitochondrial DNA; tRNA, transfer RNA. 


\section{Articles Götzet al.}

within the normal range (Figure 1e). Our patient had a combined CI/CIV deficiency with 5-10\% of residual activities, and a 50\% reduction in CIII activity in the skeletal muscle (Table 1). Blue-native electrophoresis (Figure 1f) indicated total loss of $\mathrm{CI}$ and CIV in the liver and skeletal muscle and a severe loss of both complexes in the heart as well, indicating a total inability of tRNA ${ }^{\text {Ser (UCN) }}$ to participate in mitochondrial translation. The brain showed a severely decreased amount of CIV protein, but a close to normal amount of CI.

Computational modeling showed that the transition of $\mathrm{G}>\mathrm{A}$ occurred at the highly conserved nucleotide of the acceptor (ACC) stem, occupying the last position before the start of the T-stem (Figure $1 \mathbf{b}, \mathbf{c}$ ). The mutation was predicted to lead to severe misfolding of the tRNA, with an unstable ACC stem structure. Northern blot analysis of autopsy sample-derived total RNA of the patient showed an almost total loss of fulllength tRNA ${ }^{\mathrm{Ser}(\mathrm{UCN})}$ in the heart and severe reduction in the liver and skeletal muscle (Figure 1d), together with multiple tRNA $^{\text {Ser (UCN) }}$ signals, which were not seen in the two controls. Other tRNAs that were tested for were present in the patient's tissues, indicating specific depletion of tRNA ${ }^{\text {Ser (UCN) }}$.

\section{DISCUSSION}

The patient whose case is reported here had a homoplasmic severe tRNA ${ }^{\text {Ser }(\mathrm{UCN})}$ mutation, leading to neonatal fatal lactacidosis. All previously reported mutations in tRNA ${ }^{\text {Ser (UCN) }}$ have been linked to less-severe, later-onset phenotypes, e.g., sensorineural hearing loss, progressive external ophthalmoplegia, ataxia, progressive encephalopathy, and mitochondrial myopathy, as well as MERRF/MELAS (myoclonic epilepsy with ragged red fibers/mitochondrial encephalomyopathy, lactic acidosis, and stroke-like episodes) overlap syndrome (8-12). To date, 15 disease-linked mutations have been described in this tRNA, with nine of them located either on the ACC stem or T-arm, whereas the anticodon- and D-stems allow polymorphisms. Of these pathogenic mutation positions, nine are highly conserved among 31 mammalian species (90-100\%), whereas 8 of the 10 polymorphic changes are only moderately conserved (>50\%) (13).

More than $50 \%$ of known pathogenic mtDNA mutations are clustered within the tRNA genes, which represent only $10 \%$ of the mitochondrial genome (5). Based on the results of studies in mice, this overrepresentation has been suggested to be caused by selection mechanisms that come into play during oocyte development, actively selecting against the most harmful coding-region mutations, but allowing occasional leakage and transmission of tRNA mutations of lesser severity (14-16). As the amounts of mtDNA are reduced and again amplified in oocyte development (the so-called mtDNA bottleneck) (17), mutations leaking through the surveillance mechanism may become highly amplified. In our patient's family, the absence of mutant mtDNA in the mother and again in her placenta in a subsequent pregnancy suggested that the mutation arose de novo during early oocyte development and expanded after the bottleneck, resulting in a total homoplasmic shift of mtDNA genotype within one generation.
Table 1. Mitochondrial respiratory chain activities in the skeletal muscle

\begin{tabular}{|c|c|c|c|c|}
\hline & \multicolumn{2}{|c|}{$\begin{array}{l}\text { Enzyme activity } \\
(\mathrm{nmol} / \mathrm{min} / \mathrm{mg})\end{array}$} & \multicolumn{2}{|c|}{$\begin{array}{l}\text { Enzyme activity/citrate } \\
\text { synthase activity }\end{array}$} \\
\hline & Patient & $\begin{array}{l}\text { Controls } \\
(<1 \mathrm{y})\end{array}$ & Patient & $\begin{array}{l}\text { Controls } \\
(<1 \mathrm{y})\end{array}$ \\
\hline $\begin{array}{l}\mathrm{Cl}+\mathrm{CIII} \text { (NADH: } \\
\text { cyt c reductase) }\end{array}$ & 3 & $67 \pm 45$ & 0.01 & $0.37 \pm 0.26$ \\
\hline $\begin{array}{l}\text { CII + CIII (succinate: } \\
\text { cyt c reductase) }\end{array}$ & 27 & $50 \pm 36$ & 0.05 & $0.27 \pm 0.17$ \\
\hline CII (SDH) & 26 & $24 \pm 13$ & 0.05 & $0.12 \pm 0.05$ \\
\hline CIV (COX) & 39 & $381 \pm 224$ & 0.08 & $1.92 \pm 0.55$ \\
\hline Citrate synthase & 519 & $230 \pm 122$ & - & - \\
\hline
\end{tabular}

Mitochondrial tRNAs are essential for the accurate translation of CI, CIII, CIV, and CV, and therefore mutations typically lead to combined RC deficiencies. The relatively small sequence size and strong conservation of tRNAs among species allow only few base-pair alterations in sequence without disturbing the tRNA structure and subsequent aminoacylation. Our patient had a combined CI/CIV deficiency in skeletal muscle, liver, and heart, as well as decreased levels of CIII in skeletal muscle, indicating a total inability of tRNA ${ }^{\text {Ser }(\mathrm{UCN})}$ to participate in mitochondrial translation. The newborn patient did not, however, show clinical or morphological signs of heart or liver disease; however, these would probably have developed if the patient had not died of respiratory insufficiency.

Our computational modeling predicted the patient's mutation as one leading to severe misfolding and an unstable ACC stem structure. Indeed, the autopsy samples of the patient showed an almost total specific loss of tRNA ${ }^{\text {Ser (UCN) }}$ in the heart, and severe reduction in the liver and skeletal muscle. Multiple abnormal tRNA ${ }^{\text {Ser (UCN) }}$ signals in the patient's samples suggested the presence of incompletely processed forms of the tRNA. A severe structural defect of the mutant tRNA may make it primarily unstable, or prevent binding to the mitochondrial seryl-tRNA synthetase, leading to degradation of unaminoacylated tRNA (18-20). The dramatic lack of a specific tRNA explains the severe RC deficiency, leading to uncontrolled lactic acidosis and rapid progression to fatality from the disease in this infant.

In conclusion, we consider the homoplasmic G>A 7453 mutation to be a novel pathogenic cause of neonatal lactacidosis because (i) it was not found in public databases or in Finnish mtDNA sequences; (ii) combined RC deficiency is a logical functional cause of a mitochondrial tRNA mutation; (iii) the mutation site is highly conserved among species; (iv) the mutation is predicted to affect the tRNA structure dramatically; and (v) the total RNA of the patient's tissues showed the lack of full-length tRNA ${ }^{\mathrm{Ser}(\mathrm{UCN})}$ and the presence of unprocessed forms. Heteroplasmy is typically considered to be a feature of pathogenic mtDNA mutations, and homoplasmic changes may tend to be overlooked. Our data show that 
homoplasmic de novo changes of mtDNA may underlie severe neonatal mitochondrial disorders.

\section{METHODS}

\section{Case Study of a Patient With an mtDNA Mutation}

All samples were obtained for diagnostic purposes, and were used for research only after obtaining informed consent from the parents. The study was approved by the Ethics Committee for Pediatrics, Adolescent Medicine, and Psychiatry of Helsinki University Central Hospital.

The patient we describe here was the son of healthy Finnish parents, the first child of the mother and the third of the father. His two paternal siblings were healthy, and the parents were nonconsanguineous. The baby was born at term after a normal pregnancy. He weighed 2,815g, and his Apgar scores were 3/6/8. Immediately after birth, he developed hypotonia and bradycardia, and had no spontaneous breathing. He was dependent on mechanical ventilation. He had severe lactic acidosis (up to $20 \mathrm{mmol} / \mathrm{l}$, normal value $<2.2 \mathrm{mmol} / \mathrm{l}$ ) with lactate/pyruvate ratio of 31 , elevated plasma alanine $(965 \mu \mathrm{mol} / \mathrm{l}$, normal $150-600 \mu \mathrm{mol} / \mathrm{l})$, and elevated serum creatine kinase levels (up to $3,590 \mathrm{U} / \mathrm{l}$, normal $<170 \mathrm{U} / \mathrm{l}$ ) in the blood. Urine organic acid analysis showed increased excretion of lactate, whereas blood pyruvate, hydroxybutyrate, and acylcarnitine profiling were normal. Heart, brain, and abdominal ultrasound were normal. No muscle weakness was observed; spontaneous leg movements were normal. Lactic acid was persistently high, and the patient died on day 10 due to respiratory insufficiency.

A muscle sample at the age of $5 \mathrm{~d}$ showed almost no cytochrome c oxidase activity, and mitochondrial proliferation was seen, mimicking ragged red fibers. Type 2 fiber atrophy and necrotic fibers were observed (Figure 2). At autopsy, the gross findings and weight of the heart and parenchymal organs were normal, except for the spleen and the adrenals, which were small. The macroscopic and light microscopic

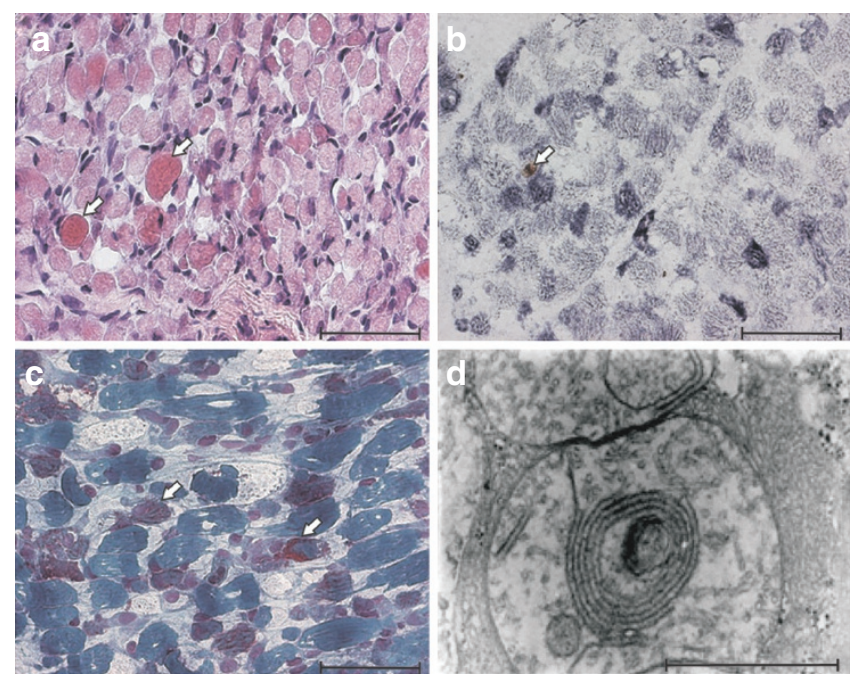

Figure 2. Morphological analysis of the skeletal muscle of the patient. (a) Hematoxylin and eosin staining of muscle tissue shows generalized severe fiber size variation and fibrosis. Large, intensively stained hypercontracting fibers (arrows) indicate tissue degeneration, $(\times 400$ original magnification, bar $50 \mu \mathrm{m}$ ). (b) Histochemical assay of frozen muscle section for cytochrome c oxidase (COX) (brown) and succinate dehydrogenase (blue) activities reveals total COX deficiency (arrow indicates one putative COX-positive fiber, $\times 400$ original magnification, bar $50 \mu \mathrm{m}$ ). (c) Modified Gomori trichrome staining shows accumulation of abnormal mitochondria in the subsarcolemmal region (ragged red fibers, arrows; $\times 400$ original magnification, bar $50 \mu \mathrm{m}$ ), and muscle fiber degeneration. (d) Ultrastructure of muscle tissue shows enlarged, swollen mitochondria with concentric crista deformations ( $\times 30,000$ original magnification, bar $1 \mu \mathrm{m})$. evaluations of the brain showed normal results. Cultured cell samples of the patient were not available for functional testing.

\section{Biochemical Analysis of RC Enzyme Activities}

The mitochondria were isolated from a fresh muscle biopsy specimen, and the RC enzyme activities, rotenone-sensitive nicotinamide adenine dinucleotide: cytochrome $\mathrm{c}$ oxidoreductase, antimycin-sensitive succinate: cytochrome c oxidoreductase (CII + CIII), succinate dehydrogenase, cytochrome c oxidase, and citrate synthase were analyzed as previously described (21).

\section{mtDNA Sequencing and Analysis}

The entire mtDNA sequence was determined by high-throughput mtDNA sequence analysis, utilizing amplification of mtDNA in two large fragments followed by DNA sequencing with several internal primers (details of primer sequences available on request), utilizing the BigDye Terminator v3.1 sequencing kit (Applied Biosystems, Warrington, UK), and the ABI 3730XL DNA Analyzer (Applied Biosystems, Foster City, CA). The revised Cambridge reference sequence of the complete human mtDNA (AC_000021, NC_012920) was used as reference sequence. Sequence analysis was carried out using Sequencher 4.5 software (GeneCodes, Ann Arbor, MI). We used the following databases to exclude known polymorphisms and reported pathogenic mutations: MITOMAP: A human mitochondrial genome database, and $\mathrm{mtDB}$ Human Mitochondrial Genome Database (http://www.mitomap.org/ MITOMAP, http://www.mtdb.igp.uu.se/).

For detection and quantification of mutant and wild-type mtDNA populations in the samples (brain, heart, liver, and skeletal muscle of the patient, blood from the mother, and a placental sample) we utilized solid-phase minisequencing as described in (22), using specific primers for the G7453A mutation. The sensitivity of the assay was $<7 \%$.

\section{mtDNA Copy-Number Quantification}

Muscle mtDNA quantification was performed using real-time quantitative PCR with TaqMan probes (Applied Biosystems, Roche, Branchburg, NJ) specific for a portion of the mitochondrial $M T-C Y B$ gene; a nuclear two-copy gene, $A P P$, was used as a reference, as previously described (23).

\section{Northern Blot Analysis}

To test for the presence of mature tRNA products, we extracted RNA from the autopsy-derived heart, skeletal muscle, and liver of the patient and of two age-matched controls. RNA was electrophoresed on a $15 \%$ $7 \mathrm{~mol} / \mathrm{l}$ urea polyacrylamide gel and blotted onto a positively charged nylon membrane (Amersham Hybond - N+, GE Healthcare Limited, Little Chalfont, UK). The RNA was UV-cross-linked and hybridized with $\left.\gamma-{ }^{32} \mathrm{P}\right]$ ATP-labeled (Perkin Elmer, Waltham, MA) oligonucleotide probes for tRNA ${ }^{\text {Ser (UCN) }}$ and three control tRNAs: tRNA ${ }^{\text {Leu (UUR), }}$ tRNA $^{\text {Gln }}$, and tRNA ${ }^{\text {Ser (AGY) }}$, and detected using a Typhoon 9400 Variable Mode Imager (Amersham Biosciences, GE Healthcare Limited).

\section{RC Protein Analysis}

Mitochondrial proteins were extracted from autopsy-derived heart, liver, brain, and skeletal muscle samples. The samples were separated on a $6-15 \%$ blue-native polyacrylamide gel by electrophoresis, and blotted onto a polyvinylidene fluoride membrane (Millipore, Billerica, MA). For protein detection, we used monoclonal antibodies (Mitosciences, Eugene, OR) against the $39 \mathrm{kDa}$ subunit of RC CI, the $70 \mathrm{kDa}-\mathrm{Ip}$ subunit of $\mathrm{CII}$, and subunit 4 of $\mathrm{CIV}$, as previously described (24).

\section{Computational tRNA Modeling}

We used computational modeling to study the consequences of the mutation on tRNA structure, using the Vienna RNAfold (Institute for Theoretical Chemistry, University of Vienna, http://rna.tbi.univie. ac.at/cgi-bin/RNAfold.cgi) and RPI mfold web server (RNA folding, The RNA Institute, University of Albany (http://mfold.rna.albany. $\mathrm{edu} / \mathrm{q}=\mathrm{mfold}$ ) programs.

\section{ACKNOWLEDGMENTS}

We would like to thank the patient's family for agreeing to participate in the study. We also thank Uwe Richter for laboratory assistance. 


\section{Articles $\mid$ Götzet al.}

\section{STATEMENT OF FINANCIAL SUPPORT}

The study was supported by grants from the Sigrid Jusélius Foundation, the Jane and Aatos Erkko Foundation, the Academy of Finland, the University of Helsinki, the Helsinki University Central Hospital (to A.S.), the Graduate School of Biotechnology and Molecular Biology, the Finnish Cultural Foundation (to A.G.), and the Maud Kuistila Memorial Foundation (to P.I.).

\section{REFERENCES}

1. Wallace DC. Mitochondrial DNA mutations in disease and aging. Environ Mol Mutagen 2010;51:440-50.

2. Schaefer AM, McFarland R, Blakely EL, et al. Prevalence of mitochondrial DNA disease in adults. Ann Neurol 2008;63:35-9.

3. Thorburn DR. Mitochondrial disorders: prevalence, myths and advances. J Inherit Metab Dis 2004;27:349-62.

4. Elliott HR, Samuels DC, Eden JA, Relton CL, Chinnery PF. Pathogenic mitochondrial DNA mutations are common in the general population. Am J Hum Genet 2008;83:254-60.

5. Florentz C, Sohm B, Tryoen-Tóth P, Pütz J, Sissler M. Human mitochondrial tRNAs in health and disease. Cell Mol Life Sci 2003;60: 1356-75.

6. Degoul F, Diry M, Rodriguez D, et al. Clinical, biochemical, and molecular analysis of a maternally inherited case of Leigh syndrome (MILS) associated with the mtDNA T8993G point mutation. J Inherit Metab Dis $1995 ; 18: 682-8$

7. Tulinius MH, Houshmand M, Larsson NG, et al. De novo mutation in the mitochondrial ATP synthase subunit 6 gene (T8993G) with rapid segregation resulting in Leigh syndrome in the offspring. Hum Genet 1995;96:290-4.

8. Bidooki S, Jackson MJ, Johnson MA, et al. Sporadic mitochondrial myopathy due to a new mutation in the mitochondrial tRNASer(UCN) gene. Neuromuscul Disord 2004;14:417-20.

9. Hutchin TP, Parker MJ, Young ID, et al. A novel mutation in the mitochondrial tRNA(Ser(UCN)) gene in a family with non-syndromic sensorineural hearing impairment. J Med Genet 2000;37:692-4.

10. Nakamura M, Nakano S, Goto Y, et al. A novel point mutation in the mitochondrial tRNA(Ser(UCN)) gene detected in a family with MERRF/ MELAS overlap syndrome. Biochem Biophys Res Commun 1995;214: 86-93.
11. Reid FM, Vernham GA, Jacobs HT. A novel mitochondrial point mutation in a maternal pedigree with sensorineural deafness. Hum Mutat 1994;3:243-7.

12. Tiranti V, Chariot P, Carella F, et al. Maternally inherited hearing loss, ataxia and myoclonus associated with a novel point mutation in mitochondrial tRNASer(UCN) gene. Hum Mol Genet 1995;4:1421-7.

13. Helm M, Brulé H, Friede D, Giegé R, Pütz D, Florentz C. Search for characteristic structural features of mammalian mitochondrial tRNAs. RNA 2000;6:1356-79.

14. Fan W, Waymire KG, Narula N, et al. A mouse model of mitochondrial disease reveals germline selection against severe mtDNA mutations. Science 2008;319:958-62.

15. Stewart JB, Freyer C, Elson JL, Larsson NG. Purifying selection of mtDNA and its implications for understanding evolution and mitochondrial disease. Nat Rev Genet 2008;9:657-62.

16. Stewart JB, Freyer C, Elson JL, et al. Strong purifying selection in transmission of mammalian mitochondrial DNA. PLoS Biol 2008;6:e10.

17. Wai T, Teoli D, Shoubridge EA. The mitochondrial DNA genetic bottleneck results from replication of a subpopulation of genomes. Nat Genet 2008;40:1484-8.

18. Toompuu M, Yasukawa T, Suzuki T, et al. The 7472insC mitochondrial DNA mutation impairs the synthesis and extent of aminoacylation of tRNASer(UCN) but not its structure or rate of turnover. J Biol Chem 2002;277:22240-50

19. Nagao A, Suzuki T, Suzuki T. Aminoacyl-tRNA surveillance by EF-Tu in mammalian mitochondria. Nucleic Acids Symp Ser (Oxf) 2007:41-2.

20. Ohtsuki T, Watanabe Y. T-armless tRNAs and elongated elongation factor Tu. IUBMB Life 2007;59:68-75.

21. Majander A, Rapola J, Sariola H, Suomalainen A, Pohjavuori M, Pihko H. Diagnosis of fatal infantile defects of the mitochondrial respiratory chain: age dependence and postmortem analysis of enzyme activities. J Neurol Sci 1995;134:95-102.

22. Suomalainen A, Syvänen AC. Quantitative analysis of human DNA sequences by PCR and solid-phase minisequencing. Mol Biotechnol 2000;15:123-31.

23. Götz A, Isohanni P, Pihko $H$, et al. Thymidine kinase 2 defects can cause multi-tissue mtDNA depletion syndrome. Brain 2008;131(Pt 11):2841-50.

24. Wittig I, Braun HP, Schägger $H$. Blue native PAGE. Nat Protoc 2006;1:418-28. 